\title{
DOS NUEVAS ESPECIES Y UNA COMBINACIÓN EN EL GÉNERO STIPA L., SECCIÓN LEIOSTIPA DUMORT. (POACEAE) PARA EL SE DE ESPAÑA
}

\author{
Francisco M. VÁZQUEZ, Hildemar SCHOLZ y Michaela SONNENTAG
}

RESUMEN. Dos nuevas especies y una combinación en el género Stipa L. seccion Leiostipa Dumort. (Poaceae) para el SE de España. En este estudio se describen dos nuevas especies (S. filabrensis y $S$. bufensis) y una combinación (S. cazorlensis) del género Stipa L., sección Leiostipa Dumort. para la flora del sudeste de la Península Ibérica. S. filabrensis es una especie endémica del macizo de Filabres y $S$. bufensis (=S. lagascae var. australis sensu F.M. Vázquez \& Devesa) está distribuida por la mitad oriental de la Península Ibérica.

Palabras clave. Poaceae, Stipa, Taxonomía, Península Ibérica.

ABSTRACT. Two new species and a combination of Stipa L. section Leiostipa Dumort. (Poaceae) genus, from SE Spain. The taxonomic study of the Stipa L. section Leiostipa Dumort genus from the Southeastern of Iberian Peninsula resulted in two new species (S. filabrensis and S. bufensis) and a combination ( $S$. cazorlensis). S. filabrensis is a endemic species from Filabres mountains (Almeria, Spain), and S. bufensis (=S. lagascae var. australis sensu F.M. Vázquez \& Devesa) is distributed from eastern of Iberian Peninsula.

Key words. Poaceae, Stipa, Taxonomy, Iberian Peninsula.

\section{INTRODUCCIÓN}

El género Stipa L. se encuentra ampliamente distribuido por todo el continente Eurasiático, contando con numerosos centro de diversificación y especiación, que lo hacen especialmente complejo por las sutiles diferencias morfológicas y ecológicas que es necesario utilizar para comprender algunas especies y describir otras nuevas.

En el cuadrante sudoccidental del Mediterráneo, la sección Leiostipa Dumort., distribuida por todo el continente Eurasiático y la mitad del norte de África, cuenta con numerosas especies endémicas como: $S$. balansae H.Scholz, S. clausa Trabut, $S$. lagascae Roemer \& Schultes, S. letournexii Trabut, S. maroccana H.Scholz, S. meridionalis F.M. Vázquez \& Devesa, o S. tunetana H.Scholz (Scholz, 1991 y 1996; Trabut, 1889; Vázquez \& Devesa, 1996 a y 1997).

La presencia de numerosos taxones endémicos, algunos con distribución muy reducida y la proximidad morfológica entre ellos, ha propiciado buen número de errores de identificación en el material recolectado. El elemento de contraste, que han servido para determinar objetivamente estos materiales es 
el tipo (lectótipo, holótipo, isótipo, etc.); difícil de conseguir en numerosas ocasiones.

Los representantes de la sección Leostipa Dumort., se caracterizan por la presencia de flores con glumas setáceas, glabras, subiguales; lema provistas de pubescencia seriada; arista bigeniculada, con pubescencia de pelos cortos, menores de $2 \mathrm{~mm}$ en las zonas angulares e interangulares; callo agudo y pubescente; pálea menor o igual que la lema, con dorso pubescente y anteras glabras o pubescentes en el ápice (fig. 1).

El objetivo de este trabajo, es dar a conocer los resultados encontrados en el estudio del material de la sección Leiostipa Dumort., procedente del sudeste de la Península Ibérica.

\section{RESULTADOS}

La revisión del material de Stipa de la Sierra de Filabres, así como el de los restantes taxones de la sección Leiostipa, representados en la Península Ibérica, ha permitido comprobar que existen dos nuevas especies para este género distribuidas en la mitad oriental de España.

El primer taxón que se va a discutir y contrastar con el resto de las especies previamente descritas es el procedente de las recolecciones de la Sierra de Filabres. Las características utilizadas para diferenciarlo del resto de las especies españolas de la sección Leiostipa, aparecen recogidas en la tabla 1.

Además de las diferencias morfológicas florales y de la lígula, es posible encontrar nuevas variaciones en relación a la distribución y dimensión de los pelos que aparecen en el dorso de la lema y en las caras abaxial y adaxial de las hojas fértiles (fig. 1).

El conjunto de diferencias encontradas en la población de la Sierra de Filabres (Almería) con respecto al resto de los taxones estudiados, permiten justificar la creación de una nueva especie:

Stipa filabrensis H. Scholz, M. Sonnentag \& F.M. Vázquez sp. nov.
Diagnosis: Differt a Stipa bufensis lemmatibus longioribus latioribusque 10,5 $11,5 \times 0,6-0,8$ (versus $8,5-10,5 \times 0,4-0,6$ ) $\mathrm{mm}$ atque pilis columnae aristae longioribus usque ad 0,5 (versus 0,2 ) $\mathrm{mm}$, a St. maroccana et omnibus speciebus sect. Leiostipae Hispaniae ligulis foliorum culmorum valde brevioribus $1,0 \mathrm{~mm}$ attingentibus.

Descripción: Tallos de hasta $70 \mathrm{~cm}$, erectos, con nudos violáceos. Hojas con vaina glabra; lígula de hasta $1 \mathrm{~mm}$, aguda, finamente escábrida; limbo de 0,7-1,5 mm de diámetro, convoluto, glabro en la cara abaxial y finamente escábrido en la cara adaxial de las hojas fértiles. Panícula de 10-20 cm, laxa, con ramas y pedúnculos finamente escábridos. Glumas glabras, con 5 nervios glabros, verdosos; la superior de (30-)32-43(-45) mm y la inferior de (32-)35-45(-48) mm. Lemas de (10-)10,5$11,5 \mathrm{~mm}$; callo de $2-3 \mathrm{~mm}$; arista de $15-18 \mathrm{~cm}$, con pelos de hasta $0,5 \mathrm{~mm}$ en la columna. Páleas de (9-)9,5-10,5 mm, con dorso pubescente. Lodículas anteriores de hasta 2 mm. Estilos 3 plumosos. Florece en Junio.

Holótipo: España: Almería, Sierra de los Filabres, below Calar alto, Piedro de Ladrones, 2050 m, 24.VI.1998, M. Sonnentag 127/14 (B); Isótipo (HSIA).

Ecología: La especie se conoce sólo de la localidad tipo, donde crece en las cumbres más altas sobre suelos silíceos, en piornales formados por Erinacea anthyllis Link y Thymus serpylloides subsp. gadorensis (Pau)Jalas.

Por otro lado el estudio detallado del material procedente del sudeste de la Península Ibérica relacionado con las especies $S$. clausa y $S$. lagascae, ha permitido comprobar que los taxones $S$. clausa subsp. cazorlensis F.M. Vázquez \& Devesa y $S$. lagascae var. australis sensu F.M. Vázquez \& Devesa (Vázquez \& Devesa, 1996 b), presentan suficientes diferencias morfológicas y ecológicas como para separarlos a nivel específico. En el caso de $S$. clausa subsp. cazorlensis, se tratan de 

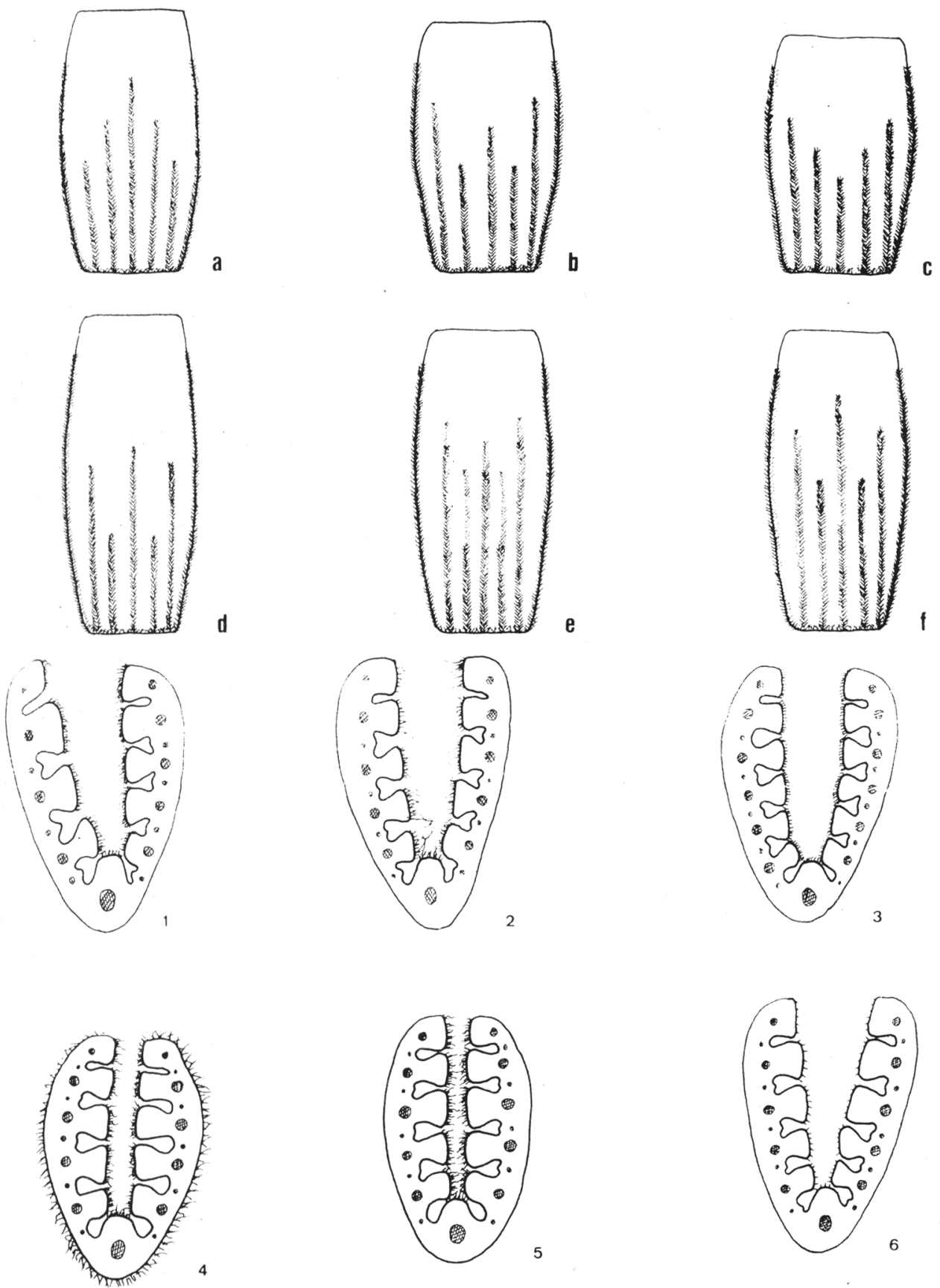

Figura 1. Distribución de los pelos en el dorso de la lema y en la cara adaxial del limbo de las hojas fértiles. Lemas de: a) S. filabrensis; b) S. bufensis; c) S. lagascae; d) S. clausa; e) S. cazorlensis y f) S. juncea. Sección del limbo de las hojas fértiles en: 1) S. cazorlensis; 2) S. clausa; 3) S. juncea; 4) S. lagascae; 5) S. bufensis y 6) S. filabrensis. 
poblaciones meridionales, distribuidas por las sierras béticas granadinas y gienenses, sin continuidad geográfica con $S$. clausa, de la que se diferencia por numerosas características morfológicas como se observa al nivel de las hojas y flores en la tabla 1 y figura 1 . Entre las diferencias más notables se pueden destacar las dimensiones de arista, lema y palea. En $S$. clausa los órganos florales son siempre de mayores dimensiones que en $S$. clausa subsp. cazorlensis. En base a la distribución y características morfológicas que separan a los dos taxones, se ha considerado combinar a nivel específico el taxon $S$ clausa subsp. cazorlensis F.M. Vázquez \& Devesa:

Stipa cazorlensis (F.M. Vázquez \& Devesa)F.M. Vázquez, H. Scholz \& M. Sonnentag stat. et comb. nov.

Stipa clausa subsp. cazorlensis F.M. Vázquez \& Devesa, Anales Jard. Bot. Madrid 54(1): 412 (1996). (basion.)

Holótipo: UNEX 14864. Isótipo: HSIA.

Finalmente, al estudiar el material identificado como S. lagascae var australis sensu F.M. Vázquez \& Devesa, procedente de la Península Ibérica y conservado en los herbarios HSIA, UNEX y MA, y contrastarlo con el material tipo de $S$. lagascae var. australis Maire (= S. maroccana $\mathrm{H}$.Scholz), se ha comprobado que las poblaciones peninsulares no se ajustan al tipo de $S$. maroccana. Las diferencias más notables encontradas entre los dos taxones están a nivel de las dimensiones de las piezas florales: $S$. maroccana ${ }^{1}$ presenta un callo de 1-1,5 mm, la arista tiene pelos en la columna de hasta 0,5 $\mathrm{mm}$ y lema de $9-10 \mathrm{~mm}$; frente a $S$. lagascae var. australis sensu F.M. Vázquez \& Devesa con callo 1,5-3 mm, pelos en la columna de hasta 0,2 mm y lema de (8-)8,5-10,5(-11) mm. Además, las poblaciones de $S$. maroccana se encuentran distribuidas exclusivamente por el cuadrante noroccidental de Africa y los ejemplares de S. lagascae var. australis sensu F.M. Vázquez \& Devesa viven en la Península Ibérica.

S. lagascae var. australis sensu F.M. Vázquez \& Devesa, es posible separarla del resto de los taxones peninsulares de la sección Leiostipa por las características de las piezas florales y de la lígula (tab. 1). Existen además diferencias en la distribución de los pelos en el dorso de la lema y en las caras abaxial y adaxial de las hojas fértiles (fig. 1). Por todo lo anterior, se ha considerado asignar categoría específica al taxón S. lagascae var. australis sensu F.M. Vázquez \& Devesa.

Stipa bufensis F.M. Vázquez, H.Scholz \& M. Sonnentag sp. nov.

Stipa lagascae var. australis sensu F.M. Vázquez \& Devesa, non Maire (1936).

Diagnosis: Habitu Stipae lagascae Roemer \& Schultes, a qua differt laminis foliorum glabris (versus pubescentis), lemmatibus (8-)8,5-10,5(11) mm (versus (7)8-10 mm) et arista (10-)12-16(-18) cm (versus (10-)11-15(-16) mm).

Species dicata horti cultor et amicissima Isidora Monterrey -"Bufa"- (1904-1976).

Descripción: Tallos de hasta $140 \mathrm{~cm}$, erectos, con nudos violáceos. Hojas con vaina glabra a escábrida; lígula de 0,5-2,5 (-3,5) mm, aguda, escábrida; limbo de 0,5-1 mm de diámetro, convoluto, glabro a escábrido en la cara abaxial y pubescente en la cara adaxial de las hojas fértiles. Panícula de 25-60(-65) cm, laxa, con ramas y pedúnculos escábridos. Glumas glabras, con 5 nervios escábridos, verdosos; la superior de (25-)28-35(-41) mm y la inferior de (28-)31-45(-50) mm. Lemas de (8-)8,5-10,5(-11) $\mathrm{mm}$; callo de 1,5-3 $\mathrm{mm}$; arista de (10-)12-16(-18) cm, con pelos de hasta 0,2 mm en la columna. Páleas de 7-9(-10) mm, con dorso pubescente. Lodículas anteriores de 0,9- 


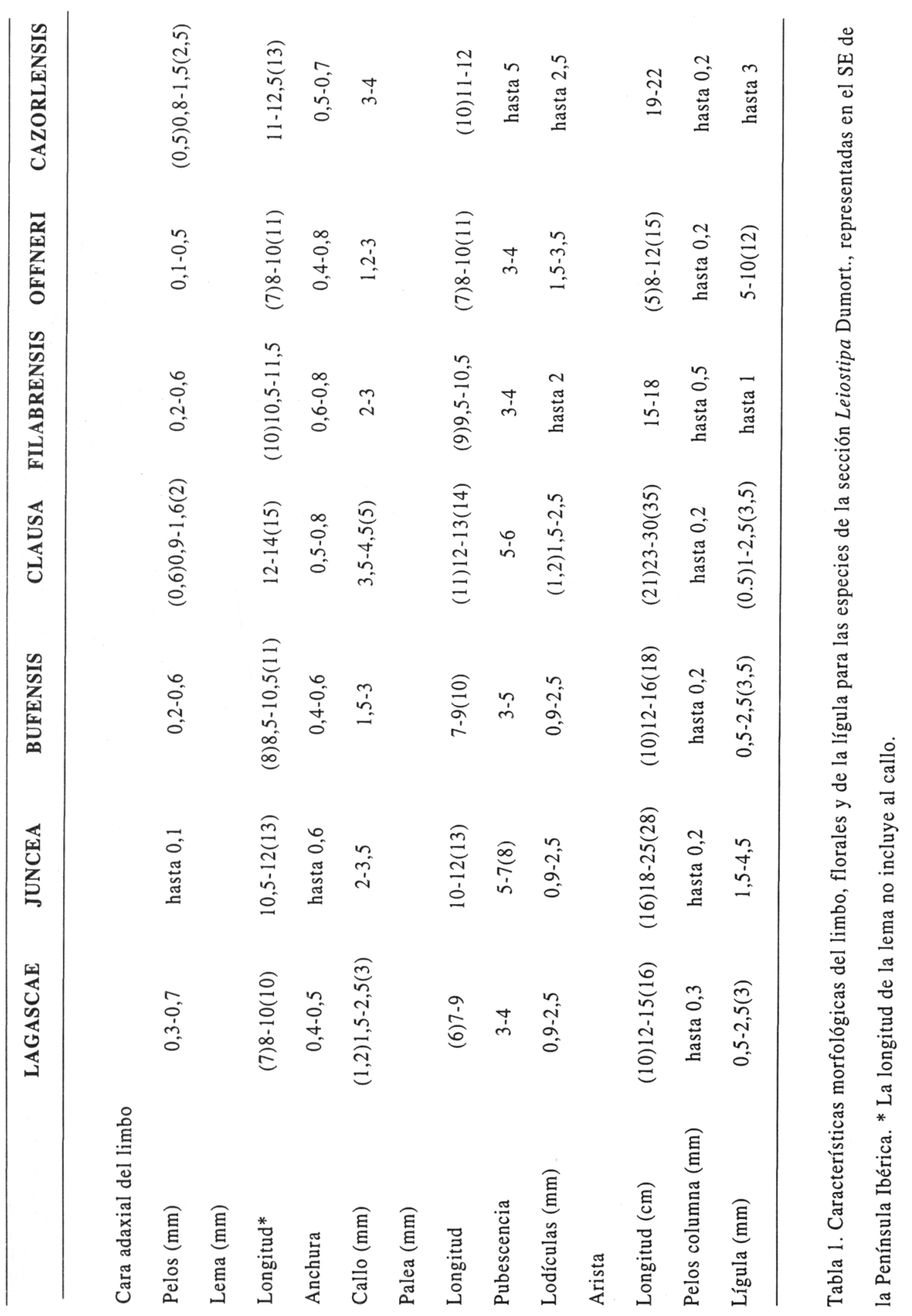


2,5 mm, la posterior de hasta 1,5 mm. Estilos 3, plumosos. Florece de Abril a Junio.

Holótipo: España: Murcia, Alhama de Murcia, 26.IV.1990, F.M. Vázquez (HSIA 3502). Isótipo (UNEX y B).

Ecología: En tomillares y lastonares subesteparios sobre suelos margosos, conviviendo con $S$. barbata Desf., y $S$. tenacissima Loef. ex L.

Distribución: SE de España (Vázquez \& Devesa 1996 b).

Clave de identificación para los taxones de la Sección Leiostipa en el SE de España

En base a las características morfológicas encontradas en este estudio, y las aportadas en estudios previos como los de Vázquez \& Devesa, 1996 a y b, se ha elaborado la siguiente clave:

1. Lígula de más de $5 \mathrm{~mm}$, aguda y glabra S. offneri

1'. Lígula de menos de $4,5 \mathrm{~mm}$, aguda u obtusa y pelosa ... 2

2. Lígula de hasta $1 \mathrm{~mm}$. Pelos en la columna de la arista de hasta $0,5 \mathrm{~mm}$ S. filabrensis

2'. Lígula de $(0,5-) 1-3(-4,5) \mathrm{mm}$. Pelos en la columna de la arista de hasta $0,3 \mathrm{~mm}$

3. Limbo de las hojas fértiles con pelos de más de 0,8 $\mathrm{mm}$ en la superficie adaxial o en el margen ........ 4

3'. Limbo de las hojas fértiles glabras o con pelos de hasta $0,3 \mathrm{~mm}$ en la superficie adaxial

4. Arista de (21-)23-30(-35) cm. Limbo de las hojas fértiles con pelos distribuidos por toda la superficie adaxial S. clausa

4'. Arista de $19-22 \mathrm{~cm}$. Limbo de las hojas fértiles con pelos distribuidos solo por los márgenes de la superficie adaxial S. cazorlensis

5. Arista de (16-)18-25(-28) cm. Limbo de las hojas fértiles con pelos de hasta $0,1 \mathrm{~mm}$......... S. juncea

5'. Arista de (10-)12-16(-18) cm. Limbo de las hojas fértiles glabras o con pelos de más de $0,2 \mathrm{~mm}$... 6

6. Limbo de las hojas con superficie abaxial pubescente. Lema de (7-)8-10 mm..S. lagascae

6'. Limbo de las hojas con superficie abaxial glabra o escábrida. Lema de (8-)8,5-10,5(-11) mm .....

S. bufensis
AGRADECIMIENTOS. Queremos expresar nuestro más sincero agradecimiento a dos correctores anónimos que con sus correcciones, anotaciones y sugerencias, mejoraron notablemente el manuscrito original.

\section{BIBLIOGRAFÍA}

MAIRE, R. -1936- Catalogue des Plantes du Maroc. Bull. Soc. Hist. Nat. Afr. Nord 24: 266.

SCHOLZ, H. -1991- Stipa tunetana, eine neue Art aus Tunesien, und das St. lagascae - Aggregat (Gramineae). Willdenowia 20: 77-80.

SCHOLZ, H. -1996- Die Taxonomie der Stipa fontanesii Parl., und S. maroccana, eine neue Art aus Marokko (Gramineae). Willdenowia 26: 225-228.

TRABUT, M.L. -1889- Révision des caractères des Stipa gigantea Lag., Lagascae R. et Sch., Letourneuxii sp. nov, Fontanesii Parlat.; Cleistogamie chez les Stipa. Bull. Soc. Bot. France 36: 404-407.

VÁZQUEZ, F.M. y J.A. DEVESA -1996 a- Stipa clausa Trabut (Stipa sect. Leiostipa Dumort., Gramineae), una especie olvidada del centro y sur de la Península Ibérica. Anal. Jard. Bot. Madrid 54(1): 407-414.

VÁZQUEZ F.M. y J.A. DEVESA - 1996 b- Revisión del género Stipa y Nassella Desv. (Poaceae) en la Península Ibérica e Islas Baleares. Acta Bot. Malacitana 21: 125-189.

VÁZQUEZ F.M. \& J.A. DEVESA -1997- Two new species and combinations of Stipa $L$. (Gramineae) from northwest Africa. Bot. Jour. Linnean Soc. 124: 201-209.

Aceptado para su publicación en Septiembre de 1999

Dirección de los autores. F. M. VÁZQUEZ: Dpto. Producción Forestal y Pastos. SIA-EXTREMADURA. Apartado 2206080 Badajoz (España). forestal @inia.es; H. SCHOLZ: Botanischer Garten und Botanisches Museum Berlin-Dahlem, Freie Universität Berlin. Königin-Luise-Str. 6-8. D-14191 Berlin (Alemania); M. SONNENTAG: Institut für Systematische Botanik und Pflanzengeographie. Freie Universität Berlin, FB 23, WE 2, Altensteinstrasse 6, D-14195. Berlin (Alemania) 